\title{
Improving Quality in Primary Care: A Model for Change
}

\author{
Dr. Jose A. PonceVega and Dr. Irene A. Williams \\ College of Management and Technology, Walden University, Minneapolis, Minnesota, USA \\ *Correspondence: Dr. Jose A. PonceVega; Email: joseponcevega@gmail.com
}

\begin{abstract}
Health care spending accounts for $17.7 \%$ of the gross domestic product in the United States, and it is expected to continue rising at an annual rate of 5.3\%. Despite high costs, health care quality lags behind other high-income countries; yet, over $70 \%$ of change initiatives fail. The purpose of this multiple case study was to explore strategies primary care leaders use for implementing quality improvement initiatives to improve patient outcomes and reduce waste in primary care facilities. The target population consisted of 3 health care leaders of 3 primary care facilities in southern California who successfully implemented quality improvement initiatives. The conceptual framework for this study was Kotter's 8-step of change management. Data were collected through face-to-face semi-structured interviews with senior health care leaders, document review, and quality reports. Patterns were identified through a rigorous process of data familiarization, data coding, and theme development and revision. Interpretations from the data were subjected to member-checking to ensure trustworthiness of the findings. Four themes emerged from this study: communication, leadership support, inclusive decision-making, and employee recognition.
\end{abstract}

KEYWORDS: Health care, Primary care, Quality, Quality improvement, Change management

\section{ARTICLE INFORMATION}

Author(s): Dr. Jose A. PonceVega and Dr. Irene A. Williams Received: 15 Aug, 2021; Accepted: 28 Aug, 2021; Published: 10 Sep, 2021; e-ISSN: 2347-4696;

Paper Id: BMN-IJBMR-2021-63;

Citation: doi.org/10.37391/IJBMR.090310

Webpage-link:

https://ijbmr.forexjournal.co.in/archive/volume-9/ijbmr-090310.html

\section{INTRODUCTION}

The United States spent $17.7 \%$ of gross domestic product (GDP) or \$3 trillion in health care expenses in 2014. Health care costs will continue to rise at a 5.3\% rate per year, and it is expected to reach a total of $19.6 \%$ of the GDP by $2024[1,2]$. The iron triangle guides the economics of health care in the United States, and cost, quality, and care comprise each side of the triangle [3]. Change initiatives in health care focus on addressing all sides by improving quality and care while decreasing cost; however, a high percentage of those change initiatives fail [4-6]. This high rate of failure in change initiatives suggests the need for research on quality improvement initiatives in primary care facilities.

Factors such as poor implementation planning, failure to create buy-in, and ineffective leadership affect implementation of quality improvement initiatives in primary care facilities [5]. In 2014, primary care visits surpassed 461 million and accounted for $52 \%$ of the total visits to health care facilities in the United States [7]. Lee, et al. [1] explained that health care cost is directly related to quality. Therefore, primary care facilities can reduce the overall cost of health care through quality improvement initiatives. The findings of this study contribute to the professional practice by offering strategies to successfully manage change and implement quality improvement initiatives that reduce waste and improve patient outcomes in primary care facilities.

\section{LITERATURE REVIEW}

\subsection{Change Management Framework}

Primary care leaders must consider various essential steps to implement successful change in an organization [8]. Kotter's [9] eight-step process developed in 1995 is well known for successful change management and organizational transformation $[10,11]$; therefore, it offered the appropriate framing for this qualitative study. Kotter's process provided a conceptual structure to explore leadership strategies for implementing quality improvement initiatives because successful changes in clinical practice must be adaptable and dynamic [10]. Focusing on quality improvement efforts in areas aligned with patients' interests create leadership and personnel buy-in, which has a positive impact on the organizational bottom line.

Kotter's eight-step model is used widely for implementing and sustaining change $[11,12]$. Kotter's process framework may assist primary care leaders in using a systematic and strategic approach to implement organizational change by connecting with people's emotions and enabling employees to identify solutions to possible problems [10]. The eight-step process includes: (a) developing a sense of urgency, (b) creating a guiding coalition, (c) developing a vision and strategy, (d) communicating the change vision, (e) empowering broadbased change, (f) generating short-term wins, (g) consolidating gains and producing more change, and (h) cultivating a culture of change.

\subsection{High Reliability in Health Care}

Pressure from government agencies, health insurance companies, and health care consumers to improve quality outcomes and reduce waste in health care organizations will continue to drive health care leaders to seek zero harm. Tolk, Cantu, and Beruvides [13] established that the concept of a high reliability organization (HRO) surfaced in 1981. HROs 
operate in hazardous environments and use work practices and behavioral procedures to attain excellence and maintain safety [13]. Industries like air traffic control, aircraft carriers, and nuclear power plants continue to operate in dangerous conditions with nearly error-free outcomes [13]. Chassin and Loeb [14] argued that primary care facilities could also achieve high reliability by engaging in change initiatives to improve quality. However, primary care leaders face challenges in pursuing high reliability because a high percentage of the change initiatives in health care organizations fail.

Chassin and Loeb [14] explained that primary care organizations seeking high reliability must engage in three domains. The three domains are leadership commitment, a culture of safety, and robust process improvement [14]. Vogus and Iacobucci [15] explained the connection of high reliability with increasing quality in health care organizations. As organizations seek to deliver failure-free health care services through leadership commitment, a culture of safety, and process improvement, quality will increase [16]. Vogus and Iacobucci [15] described the limited success in improving quality, and primary care facilities are not exempt from sharing those limitations.

\subsection{Quality in Primary Care}

Primary care is a critical tool in reaching objectives constituting the value of the overall health care system as it provides a logical basis for an efficient system. Lee, et al. [1] acknowledged that objectives constituting value in health care include the high quality of care, patient satisfaction, and the effective use of resources in the health care setting. Primary care respects the immediate needs of patients and the sense of responsibility and competence of first contact health care professionals [17]. Edwards, et al. [18] described an efficient health care system as one that involves balancing of patient needs, economic concerns, and environmental costs. It is the core responsibility of the health care practitioners and facilities to provide patients with efficient, appropriate, and humane care.

Quality in primary care refers to providing the right attention to patients at the right time while aiming at the best possible patient outcome and keeping the patient safe from any hazards or harm $[6,19]$. The primary concern of high quality care should be characterized by the ease of accessibility of services for all while addressing the health needs of patients, provision of widespread services to meet patient needs, and services centered toward the patient rather than the disease [20]. Additionally, quality care ensures coordination of care for individual patients with a holistic approach integrating psychological, biomedical, and social dimensions as well as a focus on prevention of diseases, promotion of health, and management of established health problems [20, 21]. Quality improvement in primary care provides an opportunity to focus the care to meet the patient needs.

The World Health Organization (WHO) calls on all countries to strengthen primary health care systems, improve the effectiveness of health care overall, provide better public health, keep health care costs at manageable levels, and provide equality for all to access the appropriate health care while ensuring sustainability of the health care systems [22]. van den Driessen Mareeuw, et al. [23] reiterated WHO's six dimensions of quality in primary care, and they include care being effective, efficient, accessible, patient-centered, equitable and safe. Simou, et al. [22] explained that to assess performance, WHO implemented quality health indicators of health services. Harris, et al. [24] described how improvement in the quality of care enhances accountability of managers and health care practitioners, provides resource efficiency, identification, and minimization of medical errors, while maximizing the use of adequate care, improving patient outcomes, and aligning care to specific patient needs. In fact, quality improvement in health care is the core mandate of health care settings [25]. Understanding the quality indicators will assist primary care leaders in improving overall quality and maximizing reimbursement opportunities.

\subsection{Quality Indicators}

Indicators are measurable items used as building blocks in the assessment of care. A performance evaluation is fundamental to improvement in the value of primary care and the overall health care [26]. Quality health indicators that assess primary care system performance focus on evaluating access, continuity of care, and holistic approach to care with a family and community-based orientation and coordination [22, 27]. Therefore, the quality indicators are in reaction to the multidimensional needs of patients and vital in gauging performance in primary care settings.

Leading organizations around the world, such as WHO, the Organisation for Economic Co-operation and Development (OECD), and the Agency for Healthcare Research and Quality (AHRQ) developed and implemented systems to monitor health and quality health indicators to assess the performance of health services provided at regional, national, and international level [22, 23, 28]. Simou, et al. [22] described how the 2007 National Healthcare Quality Report published 41 indicators for primary care. However, the Practice Partner Research Network (PPRNet) comprises the most useful data for primary care by utilizing an electronic medical record tool named the Accelerating the Translation of Research into Practice (A-TRIP) [22]. Both systems allow monitoring of quality measures by different agencies or stakeholders of primary care practices.

Prevention quality indicators are a set of quality procedures used in the identification of potential problems in the health care setting, following movements over time, and ascertaining differences across sections, providers, and communities [29]. Primary care focuses on services in preventive care that are helpful to manage chronic illnesses or stay healthy as a result of disease prevention services [30]. The prevention quality indicators use admission data from health care settings to evaluate instances where preventive services or better management of chronic illnesses could prevent admission cases [19, 29]. For example, inpatient data could provide 
admission information for instances where better outpatient services could avoid ambulatory situations. A diabetic patient may be admitted as a result of complications from poor illness monitoring or not getting the necessary education for selfmanagement of the condition. The prevention quality indicators would capture the admission and report the data to different stakeholders.

Several factors contribute to the hospitalization of patients, including lack of observance of the patient treatment regimen and environmental factors. However, prevention quality indicators offer a starting point to evaluate the value of structural aspects of services within communities [23]. Manzoli, et al. [29] explained that prevention quality indicators provide a clear picture of health care by identifying the needs that have not been met, checking how problems are being circumvented in outpatient settings, considering access to health care, and relating the performance of local health care systems within the communities. Prevention quality indicators also represent the present conditions of the health care system and pay particular interest in the ambulatory care, such as the prevention of both chronic diseases and acute illnesses [23, 29]. Prevention quality indicators are appreciated when calculated at the area or population levels to offer evidence about the possible problems within the community requiring further investigation.

The prevention quality indicators are used in preventing medical difficulties for both, acute ailments, and chronic conditions. Rinke, et al. [31] assessed how the indicators allow comparisons between different areas or regions over time, and they reflect on the quality of care provided in the community. Rinke, et al. [31] also explained how prevention quality indicators possess several strengths, but data users must exercise care when applying these quality indicators because variances in indicators may not clarify some disparities across regions. For example, the association between prevention quality indicators and the socioeconomic status is complex and makes it difficult to determine the quantity of the observed associations relating to access of care issues and other patient features distinct to the quality of care [31]. Primary care leaders must use prevention quality indicators with caution to establish disparities among regions.

\subsection{HEDIS Measures}

HEDIS refers to a set of standardized performance measures put in place by National Committee for Quality Assurance (NCQA) allowing comparison across health care settings [32]. HEDIS is an instrument used by the majority of America's health care entities to quantify the performance on critical dimensions of care. Health plans use HEDIS to identify areas that need improvement in health care [33]. The crucial health issues measured by HEDIS include the use of medication in asthma, control of high blood pressure, screening of breast cancer, and management of antidepressant medication among others $[32,33]$. Therefore, health care stakeholders utilize the HEDIS measures for various purposes, including reimbursement and quality improvement.
Health care plans use data from HEDIS and their results to improve quality of care and ensure quality in primary care [32]. As states and the national government move toward a health care sector focused on quality, HEDIS rates become more significant for health care plans and individual service providers $[34,35]$. The purchasers of health care services make use of these scores in the evaluation of health insurance industries and primary health care settings in making their medical decisions. The rates, therefore, act as the foundation for profiling of primary care physician as well as the choice of incentive programs.

DeVoe, et al. [36] explained how calculations for HEDIS rates derive from hybrid or administrative data. Claims or encounters data submitted to the health care plans comprises the administrative statistics, and the measures in this category include annual chlamydia screening, annual mammogram, annual Pap test among others $[34,36]$. Hybrid data, on the other hand, consists of both, medical record and administrative data. DeVoe, et al. [36] explained that records require an analysis of a randomly selected sample, or claims end up not including abstract data received for the medical records. In addition, the data in this category includes comprehensive diabetes care, immunizations, prenatal care, and childcare among others [36]. The data accuracy allows primary care leaders to establish improvement goals.

HEDIS offers benefits to various stakeholders of primary care facilities. For example, HEDIS is beneficial to the health care participants due to its ability to address consumer interests regarding quality assessment data [32, 37]. Additionally, it is considered and recognized in the U.S. as a secure method used for quality assessment in health care settings [32]. HEDIS measures ensure quality in primary care since it provides for national data comparisons and aid in the subsequent health care decisions by the various users of information.

HEDIS contains more than 40 different standardized administrative and clinical performance measures [38]. Origination of performance benchmarks for the various outcomes or quality processes in the health care setting follows the data derived from different health care plans. Therefore, the measures have a significant role in closing the gaps in the care of patients and reducing expensive acute care using preventive services [39]. The standards focus on quality improvement and value-based care across health care establishments, thus holding a critical place in helping health care providers achieve objectives related to positive patient outcome and high standards of care.

\subsection{Quality Improvement Strategies}

Quality improvement in primary care practices is essential for enhancing the health level of the population. Enhancement of patient experiences and outcomes, improvement of the services of the provider, and reduction of per capita expenses are paramount steps in quality improvement strategies [40]. Quraishi and Jordan [41] described how the efforts made to create quality in health care systems have seen health providers, insurers, quality improvement organizations, and 
delivery systems engage in primary care safety and performance. Primary care leaders looking to improve quality in their organization must focus on efficient quality improvement and safety strategies.

Primary care practices should use quality improvement orientations, which seek continuous improvement of the outcomes of patients and their performances. Abdallah [42] described orientation as one of the drivers of quality initiatives because it guides primary care practices in setting priorities in areas requiring improvement of the strategies to achieve quality improvement goals. Quality improvement efforts will determine the specific areas of practice to address, and the methods that will be used to deal with the particular issues. The choice of practices and the methods used to improve these aspects will vary based on the facility, circumstances, and the resources allocated for the exercise [43]. Typical areas that need improvement include identification of patients, monitoring and following up of patients with diabetes, and ensuring growth in delivery of recommended prevention services for all patients.

Quality improvement in primary care is a new activity to many health care facilities. The need to utilize new skills to meet quality improvement goals is essential [44]. The methods to improve quality include identification of areas for improvement, studying the available data to understand current situations in health care practices, planning and initiating change, and monitoring the performances through time. Silver, et al. [6] described the need to use performance boards to display a commitment to quality improvement. Solberg, et al. [45] explained how external support might be required while undertaking a quality improvement strategy. The additional support can assist in carrying out quality improvement.

Primary care systems adopted different strategies to accelerate improvement in quality. The most commonly referred strategies are Lean Principles and Six Sigma Principles. The Six Sigma approach is a quality improvement management strategy that seeks to improve efficiency [42]. While Lean strategies focus on process efficiency and waste reduction, the Six Sigma principles focus on reducing process variation [46, 47]. Six Sigma creates a unique infrastructure of persons within the primary care organization who are experts in improving quality and reducing waste.

Stakeholders in health care, the private, and governmental sectors should participate in providing support for implementing changes in the quality of primary care. External support within the hospital setup can efficiently assist primary care practitioners by providing work facilitation and coaching [48]. External facilitators help the primary care practitioners to improve their approach toward quality improvement and developing skills. Hudson, et al. [49] described how external facilitators also provide expertise and quality improvement tools, thus enabling the participants to troubleshoot challenges and barriers to implementing quality improvement in primary care. Coaching allows the practitioners to adapt to the new ways of doing business [50]. Facilitation and coaching assist primary care leaders in developing internal capacity for activities related to quality improvement.

Peer-to-peer mentoring and consultation by experts provide primary care practitioners with knowledge from experts outside their sphere of activity. Lessard, et al. [51] explained how such experience facilitates new implementations in the facility. In addition, benchmarking and the provision of feedback to the primary care practice allow obtaining the information on quality improvement performance in comparison with regional and national averages, which are essential in achieving quality improvement [22]. Feedback data will assist the teams in processing information on important indicators of processes and the outcomes regarding services, costs, experience, and patient quality.

Health care plans should create a community in which stakeholders and practitioners can share learning experiences. The community strengthens the culture of continuous quality improvement [52]. In addition, Makary and Daniel [53] indicated how communities and entities would support quality improvement in primary care by sharing best practices, lessons learned, challenges encountered, and enhancing inspiration. Having a sense of community rather than individual practices allows other organizations to lead parallel initiatives for health and care in the same area.

\section{OBJECTIVES OF THE STUDY}

\subsection{Research Purpose}

The purpose of this qualitative multiple case study was to explore strategies primary care leaders use for implementing quality improvement initiatives to improve patient outcomes and reduce waste in primary care facilities. The target population consisted of health care leaders of three primary care facilities who successfully implemented quality improvement initiatives.

\subsection{Research Question}

What strategies do some primary care leaders use for implementing quality improvement initiatives to improve patient outcomes and reduce waste in primary care facilities?

\section{RESEARCH METHODOLOGY}

\subsection{Research Design}

A case study was appropriate for this study because the intent was to explore successful strategies utilized by primary care leaders during the implementation of quality improvement initiatives. Tumele [54] utilized case study design to explore in detail a program, event, or process and develop historical explanations that can be generalized to explain other events. To achieve data saturation, researchers must reach a point of conceptual depth that allows them to theorize [55]. Although the number of participants in the study was limited to three primary care facilities, the participants had the breadth of knowledge and experience to address the research questions. In addition, document review established that no new themes emerged from the study data. 


\subsection{Sampling}

Researchers conducting qualitative research use purposeful sampling to identify participants rich in information [56]. Elo, et al. [57] and Malterud, Siersma, and Guassora [58] explained that sample sizes for qualitative studies could be small when the study aim is narrow, and the analysis includes longitudinal in-depth exploration. Nelson [55] added that sample size should not focus on the number of participants, but in the depth of the data; therefore, we emphasized on the concept of data saturation. Nelson [55] described data saturation as the point where no additional themes emerge from the data. We used this concept as a tool to determine if there was a need for additional participants for the study.

Current guidelines for thematic analysis in qualitative research suggest a sample of two to 10 participants for finding sufficient themes of the desired prevalence [59]. The population of this study consisted of three senior health care managers from primary care facilities in Southern California who have successfully implemented quality improvement initiatives. The eligibility criteria for the senior health care manager consisted of (a) being 21 years of age or older, (b) being employed by a primary care facility in Southern California, and (c) having experience with successful implementation of quality improvement initiatives in primary care. Morse, Lowery, and Steury [60] described how purposive sampling provides an opportunity to select participants who meet the criteria to answer the interview questions. Elo, et al. [57] also added that researchers interested in participants who have the most knowledge on the research topic could use purposive sampling. Utilizing purposive sampling was appropriate for this study because we selected a specific group of participants to seek specific knowledge.

\subsection{Limitations}

According to Dennison, et al. [61] and Helmich, et al. [62], limitations influence the strength of the study because they are weaknesses that researchers cannot control. The first limitation identified in this study was that the sample size of three organizations might not represent organizations in other regions. Another limitation was the participants' personal biases regarding success or failure of quality improvement initiatives. The third limitation was that the results might not transfer to other industries.

\section{RESULTS}

From the interviews and additional supporting documents, it is evident that a demand for primary care leaders to improve patient outcomes and reduce waste exists. The research also revealed four themes: (a) communication, (b) leadership support, (c) inclusive decision-making, and (d) employee recognition. Fleming, et al. [63] described how external pressures, such as the Affordable Care Act, proliferate attempts to contain cost. Focusing on sustainable initiatives will strengthen and improve quality of primary care practices. Table 1 contains a summary of the main key terms that formed patterns for the major themes. These major themes provide answers to the research question and relate to the successful implementation of quality improvement initiatives in primary care.

\begin{tabular}{|l|c|}
\hline Major Themes & Key Terms of Phrases \\
\hline Theme 1: Communication & $\begin{array}{c}\text { meeting, call, information, } \\
\text { communication, face-to- } \\
\text { face, email }\end{array}$ \\
\hline Theme 2: Leadership Support & $\begin{array}{c}\text { lead, champion, } \\
\text { management, accountable, } \\
\text { authority }\end{array}$ \\
\hline $\begin{array}{l}\text { Theme 3: Inclusive Decision- } \\
\text { Making }\end{array}$ & $\begin{array}{c}\text { team, staff, involved, } \\
\text { feedback, roles, providers, } \\
\text { contribute }\end{array}$ \\
\hline $\begin{array}{l}\text { Theme 4: Employee } \\
\text { Recognition }\end{array}$ & accolades, recognize \\
\hline
\end{tabular}

Table 1: Thematic data groups.

\subsection{Communication}

Communication emerged as a theme from the semistructured interviews and documents provided by the participants. The data analysis of the interviews revealed that communication is an important component of implementing quality improvement initiatives. Baxter, et al. [64] discussed how developing effective horizontal and vertical communication pathways prior to change implementation promote team collaboration, effectiveness, and efficiency. Table 2 includes the key terms participants used to refer to communication. Participants mentioned communication key terms a total of 99 times during the interviews.

\begin{tabular}{|l|c|}
\hline Reference & Frequency \\
\hline Meeting & 34 \\
\hline Call & 27 \\
\hline Information & 12 \\
\hline Communication & 11 \\
\hline Email & 8 \\
\hline Face-to-face & 7 \\
\hline
\end{tabular}

Table 2: References to communication.

Participants described vertical communication as a key element of successful quality improvement implementation. Saruhan [65] explained that vertical communication flows downward or upward. Downward communication was the first type described by participants because leaders informed the employees of the change and shared the importance of the initiative. Participants agreed that it was imperative to success to have a face-to-face meeting with employees for the first notification of change.

Upward communication is another type of vertical communication, and participants shared that during meetings, leaders encouraged staff to share information with leadership to make decisions based on their inputs. Participants also stated that expert deference was important because employees are the most knowledgeable in their respective areas; therefore, leaders needed to listen to employees' input and make decisions based on the feedback from the experts. One participant shared that horizontal communication motivated team members to contribute to success because discussion 
generated a sense of competition because employees did not want to be outperformed by their peers.

\subsection{Leadership Support}

The second theme that emerged from the data analysis indicated that leadership support is an imperative consideration for successful quality improvement initiatives. All participants referenced the support of the organizational leaders during the interviews. Key terms describing leadership support appeared 34 times in the data analysis as demonstrated in Table 3.

\begin{tabular}{|l|c|}
\hline Reference & Frequency \\
\hline Lead & 14 \\
\hline Management & 9 \\
\hline Champion & 8 \\
\hline Authority & 2 \\
\hline Accountable & 1 \\
\hline
\end{tabular}

Table 3: References to leadership support.

Participants expressed how leadership support is necessary during several steps of the quality improvement initiative. One participant attributed part of their successful initiative to the assignment of a sponsor from the c-suite. Two of the participants referenced leadership helping remove barriers during the quality improvement initiative. Understanding of the barriers that hinder implementation of change is an important factor as it helps select a guiding teamwork whose members are from diverse organizational backgrounds characterized by different expertise, credibility, and position $[66,67]$. The team to implement change should know how the organization operates and improve the communication with other stakeholders including other nurses, physician, and support staff.

Leadership support also increased commitment from employees at each of the participating facilities. One participant cited increased staff commitment to the quality improvement initiative when the leaders provided support with removing barriers to success. Leadership should be visible in supporting the people within the organization [6]. Leading by example is a technique leaders can utilize to convince employees of the need to change and continue to increase commitment from the employees.

\subsection{Inclusive Decision-Making}

Data analysis from the interviews revealed inclusive decisionmaking as the third theme of the research study. Inclusive decision-making refers to employees having input regarding proposed change [68]. Table 4 shows that inclusive decisionmaking was the most notable theme of the findings. Participants mentioned key terms of inclusive decision-making 124 instances.

\begin{tabular}{|l|c|}
\hline Reference & Frequency \\
\hline Team & 61 \\
\hline Staff & 40 \\
\hline Involved & 12 \\
\hline Roles & 8 \\
\hline
\end{tabular}

\begin{tabular}{|l|c|}
\hline Participant & 3 \\
\hline
\end{tabular}

Table 4: References to inclusive decision-making.

All participants noted the relevance of inclusive decisionmaking during the interviews. This finding aligns with a research proposed by Abrams, et al. [21], which considered inclusive decision-making as a quality benchmark. Participation allows employees to voice concerns and provide input. Ultimately participation creates a sense of fairness and respect [68]. One participant referred to inclusive decisionmaking and participation as an opportunity to empower employees and make them feel invested in the initiative. Another participant added that employees are often the subject matter experts; therefore, participation is essential for success of the initiative.

\subsection{Employee Recognition}

Employee recognition emerged as the fourth theme of the data analysis. When an organization makes an effort to reward and recognize employees, the employees reciprocate by fully engaging in their roles and responsibilities [69]. Table 4 shows that participants cited key terms of employee recognition a total of 11 times. All participants discussed this theme during their interviews.

\begin{tabular}{|l|c|}
\hline Reference & Frequency \\
\hline Recognize & 7 \\
\hline Accolades & 4 \\
\hline
\end{tabular}

Table 5: References to employee recognition.

Staff recognition is a tool leadership can use to increase staff engagement during quality improvement initiatives. As noted by many researchers, employee recognition affects job satisfaction and commitment to the organization [70]. Another aspect of employee recognition discussed during the interviews was the form of recognition or reward. Rewards and recognition take many forms, and leaders must ensure that the program is in line with the goals and objectives of the facility [71]. Kosfeld, Neckermann, and Yang [72] presented financial incentives and nonfinancial incentives as two types of employee recognition. Participants mentioned examples of both types of employee recognition.

\section{FURTHER RESEARCH}

The purpose of this qualitative multiple case study was to explore strategies primary care leaders use for implementing quality improvement initiatives to improve patient outcomes and reduce waste in primary care facilities. The population consisted of health care leaders of three primary care facilities in Southern California who successfully implemented quality improvement initiatives. Helmich, et al. [62] described how limitations are weaknesses of the study. There were two limitations identified in this study that could be addressed with further research. They include the sample size and transferability to other industries.

According to the California Health and Human Services [73], there are 1,359 primary care clinics in the state. Since I included only three primary care facilities, adjusting the 
sample size for a larger number could impact the results. The study could have a different outcome by utilizing a larger sample size; therefore, the study warrants additional research of sustainability strategies primary care leaders could use for improving quality and reducing waste. The study also focused on primary care leaders that implemented quality improvement initiatives. It may be beneficial to explore the perspective of executive leadership or other employees within the primary care realm regarding quality improvement and its effect on cost, quality, and care.

Researchers should follow this study with a quantitative research. Park and Park [74] explained that qualitative studies play a crucial role in research discovery, and quantitative studies are excellent for justification of the findings. Conducting quantitative research may provide different implementation strategies of quality improvement initiatives. Understanding the frequency of the strategies discovered in this study such as communication or recognition may provide the potential correlation with outcomes.

\section{CONCLUSION}

The results of the data analysis from this study provide insightful information for primary care leaders to implement initiatives that improve patient outcomes and reduce waste in primary care facilities. The findings are applicable within the health care environment. McFadden, Stock, and Gowen [75] also explained the relationship between improving quality, reducing waste, and increasing patient safety, which is an urgent national concern due to unnecessary errors and high cost. This study offers primary care leaders an opportunity to make a significant positive impact increasing patient safety and reducing health care costs by using the strategies identified by participants to improve quality and reduce waste.

Primary care leaders may consider recommendations based on this research study to assist with implementing quality improvement initiatives geared toward improving patient outcomes and reducing waste. The study findings indicate that action steps could be beneficial when using innovative models of care to provide quality care in primary care settings. The recommendations flow logically from the conclusions and contain several action steps that leaders may incorporate in their efforts to improve quality of care. The recommendations for action are the following: (a) communicate the purpose of the initiative and its value toward quality improvement, (b) establish a leadership presence to show support and remove barriers, (c) institute inclusive decision-making through input and feedback, and (d) employ a rewards and recognition program that aligns with the objectives of the organization.

The first recommendation of this research is to communicate the purpose of the initiative and its value toward quality improvement. The importance of vertical and horizontal communication was evident in the findings. Participants also expressed the urge to use downward and upward communication in the organization. By using different communication techniques, participants were successful at implementing quality improvement initiatives.
The second recommendation is to establish a leadership presence to show support and remove barriers. All the participants cited the need for leaders to be present and assist employees with removing barriers that could jeopardize success of the initiative. Barriers discussed by participants included staffing shortages and support needed from other areas not involved in the initiative. Leadership support also increased employee commitment to the initiative and the organization.

Inclusive decision-making through input and feedback emerged as the third recommendation. Brainstorming sessions helped leaders encourage input from the employees. Additionally, including employees in the process of constructing the change and giving them opportunities to express concerns influenced outcomes of the initiative. Employee inclusion when making decisions surfaced as an integral part of quality improvement initiative success.

The last recommendation is to employ a rewards and recognition program that aligns with the objectives of the organization. Each participant shared their experience with rewards and recognitions and noted the importance of having a program. Two types of rewards that materialized from the findings were financial and nonfinancial rewards. Rewards and recognition of employees was imperative to success of the quality improvement initiative.

\section{REFERENCES}

[1] Lee, V. S., Kawamoto, K., Hess, R., Park, C., Young, J., et al. (2016). Implementation of a value-driven outcomes program to identify high variability in clinical costs and outcomes and association with reduced cost and improved quality. Journal of American Medical Association, 316, 1061-1072.

[2] Martin, A. B., Hartman, M., Benson, J., \& Catlin, A. (2016). National health spending in 2014: Faster growth driven by coverage expansion and prescription drug spending. Health Affairs, 35, 150-160.

[3] Riggs, K. R. (2015). The health care "iron triangle." Cleveland Clinic Journal of Medicine, 82, 332.

[4] Donnelly, L. F. (2017). Avoiding failure: Tools for successful and sustainable quality-improvement projects. Pediatric Radiology, 47, 793-797.

[5] Longenecker, C. O., \& Longenecker, P. D. (2014). Why hospital improvement efforts fail: A view from the front line. Journal of Healthcare Management / American College of Healthcare Executives, 59, 147-157.

[6] Silver, S. A., McQuillan, R., Harel, Z., Weizman, A. V, Thomas, A., et al. (2016). How to sustain change and support continuous quality improvement. Clinical Journal of the American Society of Nephrology, 11, 916-924.

[7] Center for Disease Control and Prevention. (2016). National Ambulatory Medical Care Survey: 2014 State and National Summary Tables.

[8] Williamsson, A., Eriksson, A., \& Dellve, L. (2016). Organization of change agents during care process redesign in Swedish health care. Journal of Hospital Administration, 5(3), 20-32. 
[9] Kotter, J. P. (1995). Leading change: Why transformation efforts fail. Harvard Business Review, 2, 59-67.

[10] Burden, M. (2016). Using a change model to reduce the risk of surgical site infection. British Journal of Nursing, 25, 949-954.

[11] Pollack, J., \& Pollack, R. (2015). Using Kotter's eight stage process to manage an organisational change program: Presentation and practice. Systemic Practice and Action Research, 28, 51-66.

[12] Hughes, M. (2016). Leading changes: Why transformation explanations fail. Leadership, 12, 449-469.

[13] Tolk, J. N., Cantu, J., \& Beruvides, M. (2015). High reliability organization research: A literature review for health care. Engineering Management Journal, 27, 218-237.

[14] Chassin, M. R., \& Loeb, J. M. (2013). High-reliability health care: Getting there from here. Milbank Quarterly, 91, 459-490.

[15] Vogus, T. J., \& Iacobucci, D. (2016). Creating highly reliable health care: How reliability-enhancing work practices affect patient safety in hospitals. Industrial and Labor Relations Review, 69, 911-938.

[16] Griffith, J. R. (2015). Understanding high-reliability organizations: Are Baldrige recipients models? Journal of Healthcare Management, 60, 44-61.

[17] Fleiszer, A. R., Semenic, S. E., Ritchie, J. A., Richer, M. C., \& Denis, J. L. (2015). The sustainability of healthcare innovations: A concept analysis. Journal of Advanced Nursing, 71, 14841498 .

[18] Edwards, S. T., Bitton, A., Hong, J., \& Landon, B. E. (2014). Patient-centered medical home initiatives expanded in 20092013: Providers, patients, and payment incentives increased. Health Affairs, 33, 1823-1831.

[19] Van Loenen, T., Faber, M. J., Westert, G. P., \& Van Den Berg, M. J. (2016). The impact of primary care organization on avoidable hospital admissions for diabetes in 23 countries. Scandinavian Journal of Primary Health Care, 34, 5-12.

[20] Bodenheimer, T., Ghorob, A., Willard-Grace, R., Grumbach, K., \& Care, U. C. (2014). The 10 building blocks of primary care forming stable teamlets. Annals of Family Medicine, 12, 166171.

[21] Abrams, M., Nuzum, R., Zezza, M., Ryan, J., Kiszla, J., et al. (2015). The Affordable Care Act's payment and delivery system reforms: A progress report at five years. Issue Brief (Commonwealth Fund), 12, 1-16.

[22] Simou, E., Pliatsika, P., Koutsogeorgou, E., \& Roumeliotou, A. (2015). Quality indicators for primary health care: A systematic literature review. Journal of Public Health Management and Practice, 21(5), E8-E16.

[23] van den Driessen Mareeuw, F. A., Hollegien, M. I., Coppus, A. M. W., Delnoij, D. M. J., \& De Vries, E. (2017). In search of quality indicators for Down syndrome healthcare: A scoping review. BMC Health Services Research, 17, 284-297.

[24] Harris, S. B., Green, M. E., Brown, J. B., Roberts, S., Russell, G., et al. (2015). Impact of a quality improvement program on primary healthcare in Canada: A mixed-method evaluation. Health Policy, 119, 405-416.

[25] Sibthorpe, B., Agostino, J., Coates, H., Weeks, S., Lehmann, D., et al. (2017). Indicators for continuous quality improvement for otitis media in primary health care for Aboriginal and Torres Strait Islander children. Australian Journal of Primary Health, 23, 1-9.
[26] Young, R. A., Roberts, R. G., \& Holden, R. J. (2017). The challenges of measuring, improving, and reporting quality in primary care. Annals of Family Medicine, 15(2), 175-182.

[27] Saust, L. T., Monrad, R. N., Hansen, M. P., Arpi, M., \& Bjerrum, L. (2016). Quality assessment of diagnosis and antibiotic treatment of infectious diseases in primary care: A systematic review of quality indicators. Scandanavian Journal of Primary Health Care, 34, 258-266.

[28] Pavlič, D. R., Sever, M., Klemenc-Ketiš, Z., \& Švab, I. (2015). Process quality indicators in family medicine: results of an international comparison. BMC Family Practice, 16(172), 1-11.

[29] Manzoli, L., Flacco, M. E., De Vito, C., Arcà, S., Carle, F., et al. (2014). AHRQ prevention quality indicators to assess the quality of primary care of local providers: A pilot study from Italy. European Journal of Public Health, 24, 745-750.

[30] Grace, S. L., Poirier, P., Norris, C. M., Oakes, G. H., Somanader, D. S., et al. (2014). Pan-Canadian development of cardiac rehabilitation and secondary prevention quality indicators. Canadian Journal of Cardiology, 30, 945-948.

[31] Rinke, M. L., Abdullah, F., Colantuoni, E., Zhang Y., Miller R., M., et al. (2015). State-mandated hospital infection reporting is not associated with decreased pediatric health care-associated infections. Journal of Patient Safety, 11, 123-134.

[32] Trivedi, A. N., Wilson, I. B., Charlton, M. E., \& Kizer, K. W. (2016). Agreement between HEDIS performance assessments in the VA and Medicare Advantage: Is quality in the eye of the beholder? A Journal of Medical Care Organization, Provision and Financing, 53, 1-3.

[33] Hu, J., Schreiber, M., Jordan, J., George, D. L., \& Nerenz, D. (2018). Associations between community sociodemographics and performance in HEDIS quality measures: A study of 22 medical centers in a primary care network. American Journal of Medical Quality, 33, 5-13.

[34] Harris, A., Ellerbe, L., Phelps, T. E., Finney, J. W., Bowe, T., et al. (2015). Examining the specification validity of the HEDIS quality measures for substance use disorders. Journal of Substance Abuse Treatment, 53, 16-21.

[35] Robst, J., Rost, K., \& Marshall, D. (2013). Do employers know the quality of health care benefits they provide? Use of HEDIS depression scores for health plans. Psychiatric Services, 64, 1134-1139.

[36] DeVoe, J. E., Huguet, N., Likumahuwa-Ackman, S., Angier, H., Nelson, C., et al. (2015). Testing health information technology tools to facilitate health insurance support: A protocol for an effectiveness-implementation hybrid randomized trial Implementation Science, 10, 1-9.

[37] Pawlson, L. G., Scholle, S. H., \& Powers, A. (2007). Comparison of administrative-only versus administrative plus chart review data for reporting HEDIS hybrid measures. American Journal of Managed Care, 13, 553-558.

[38] National Committee for Quality Assurance. (2018). HEDIS 2018 Measures.

[39] Rosenthal, M., Sinaiko, A., Eastman, D., Chapman, B., \& Partridge, G. (2015). Impact of the Rochester medical home inititiative on primary care practices, quality, utilization, and costs. Medical Care, 53, 967-973.

[40] Harvey, G., \& Lynch, E. (2017). Enabling continuous quality improvement in practice: The role and contribution of facilitation. Frontiers in Public Health, 5, 1-5. 
[41] Quraishi, J., \& Jordan, L. (2015). Quality and performance measurement: National efforts to improve quality of care through measurement development. American Association of Nurse Anesthetists, 82, 184-188.

[42] Abdallah, A. (2014). Implementing quality initiatives in healthcare organizations: Drivers and challenges. International Journal of Health Care Quality Assurance, 27, 166-181.

[43] Van der Biezen, M., Derckx, E., Wensing, M., \& Laurant, M. (2017). Factors influencing decision of general practitioners and managers to train and employ a nurse practitioner or physician assistant in primary care: A qualitative study. BMC Family Practice, 18, 1-10.

[44] Renedo, A., \& Marston, C. (2015). Spaces for citizen involvement in healthcare: An ethnographic study. Sociology, 49, 488-504.

[45] Solberg, L. I., Crain, a L., Tillema, J. O., Fontaine, P. L., Whitebird, R. R., et al. (2014). Challenges of medical home transformation reported by 118 patient-centered medical home (PCMH) leaders. Journal of the American Board of Family Medicine, 27, 449-457.

[46] Basta, Y. L., Zwetsloot, I. M., Klinkenbijl, J. H. G., Rohof, T., Monster, M. M. C., et al. (2016). Decreasing the dispatch time of medical reports sent from hospital to primary care with Lean Six Sigma. Journal of Evaluation in Clinical Practice, 22, 690-698.

[47] Ha, C., McCoy, D. A., Taylor, C. B., Kirk, K. D., Fry, R. S., et al. (2016). Using Lean Six Sigma methodology to improve a mass immunizations process at the United States Naval Academy. Military Medicine, 181, 582-588.

[48] Scott, C. P. R., Jiang, H., Wildman, J. L., \& Griffith, R. (2018). The impact of implicit collective leadership theories on the emergence and effectiveness of leadership networks in teams. Human Resource Management Review 28, 464-481.

[49] Hudson, S. M., Hiott, D. B., Cole, J., Davis, R., Egan, B. M., et al. (2014). Increasing capacity for quality improvement in underresourced primary care settings. Quality Management in Health Care, 23, 155-162.

[50] Crouzet, B., Parker, D., \& Pathak, R. (2014). Preparing for productivity intervention initiatives. International Journal of Productivity and Performance Management, 63, 946-959.

[51] Lessard, S., Bareil, C., Lalonde, L., Duhamel, F., Hudon, E., et al. (2016). External facilitators and interprofessional facilitation teams: A qualitative study of their roles in supporting practice change. Implementation Science, 11(97), 1-13.

[52] Thomas, P. (2017). Collaborating sites for community-oriented integrated care and health promotion. London Journal of Primary Care, 9, 2-6.

[53] Makary, M. A., \& Daniel, M. (2016). Medical error-the third leading cause of death in the US. British Medical Journal, 353, i2139.

[54] Tumele, S. (2015). Case study research. International Journal of Sales, Retailing and Marketing, 4(9), 68-78.

[55] Nelson, J. (2016). Using conceptual depth criteria: Addressing the challenge of reaching saturation in qualitative research. Qualitative Research (Online).

[56] Palinkas, L. A., Horwitz, S. M., Green, C. A., Wisdom, J. P., Duan, N., et al. (2015). Purposeful sampling for qualitative data collection and analysis in mixed method implementation research. Administration and Policy in Mental Health and Mental Health Services Research, 42, 533-544.
[57] Elo, S., Kaariainen, M., Kanste, O., Polkki, T., Utriainen, K., et al. (2014). Qualitative content analysis: A focus on trustworthiness. SAGE Open, 4, 1-10.

[58] Malterud, K., Siersma, V. D., \& Guassora, A. D. (2015). Sample size in qualitative interview studies: Guided by information power. Qualitative Health Research, 26(3), 1-8.

[59] Fugard, A., \& Potts, H. (2015). Supporting thinking on sample sizes for thematic analyses: A quantitative tool. International Journal of Social Research Methodology, 18, 669-684.

[60] Morse, W. C., Lowery, D. R., \& Steury, T. (2014). Exploring saturation of themes and spatial locations in qualitative public participation geographic information systems research. Society \& Natural Resources, 27, 557-571.

[61] Dennison, L., Morrison, L., Conway, G., \& Yardley, L. (2013). Opportunities and challenges for smartphone applications in supporting health behavior change: Qualitative study. Journal of Medical Internet Research, 15, 86.

[62] Helmich, E., Boerebach, B. C. M., Arah, O. A., \& Lingard, L. (2015). Beyond limitations: Improving how we handle uncertainty in health professions education research. Medical Teacher, 37, 1043-1050.

[63] Fleming, N. S., da Graca, B., Ogola, G. O., Culler, S. D., Austin, J., et al. (2017). Costs of transforming established primary care practices to patient-centered medical homes (PCMHs). The Journal of the American Board of Family Medicine, 30, 460471.

[64] Baxter, P., Cleghorn, L., Alvarado, K., Cummings, G., Kennedy, D., et al. (2016). Quality-based procedures in Ontario: Exploring health-care leaders' responses. Journal of Nursing Management, 24, 50-58.

[65] Saruhan, N. (2014). The role of corporate communication and perception of justice during organizational change process. Business and Economics Research Journal, 5, 143-166.

[66] D'Innocenzo, L., Mathieu, J. E., \& Kukenberger, M. R. (2016). A meta-analysis of different forms of shared leadership-team performance relations. Journal of Management, 42, 1964-1991.

[67] Mathieu, J. E., Tannenbaum, S. I., Donsbach, J. S., \& Alliger, G. M. (2014). A review and integration of team composition models: Moving toward a dynamic and temporal framework. Journal of Management, 40, 130-160.

[68] Rogiest, S., Segers, J., \& van Witteloostuijn, A. (2018). Matchmaking in organizational change: Does every employee value participatory leadership? An empirical study. Scandinavian Journal of Management, 34, 1-8.

[69] Downey, S. N., van der Werff, L., Thomas, K. M., \& Plaut, V. C. (2015). The role of diversity practices and inclusion in promoting trust and employee engagement. Journal of Applied Social Psychology, 45, 35-44.

[70] Ramdhani, A., Ramdhani, M. A., \& Ainisyifa, H. (2017). Model conceptual framework of corporate culture influenced on employees commitment to organization. International Business Management, 11, 826-830.

[71] Bakotić, D., \& Rogošić, A. (2017). Employee involvement as a key determinant of core quality management practices. Total Quality Management and Business Excellence, 28, 1209-1226.

[72] Kosfeld, M., Neckermann, S., \& Yang, X. (2017). The effects of financial and recognition incentives across work contexts: The role of meaning. Economic Inquiry, 55, 237-247. 
[73] California Health and Human Services. (2018). 2017 Preliminary Primary Care Clinic Annual Utilization Data.

[74] Park, J., \& Park, M. (2016). Qualitative versus quantitative research methods: Discovery or justification? Journal of Marketing Thought, 3(10), 1-7.
[75] McFadden, K. L., Stock, G. N., \& Gowen, C. R. (2015). Leadership, safety climate, and continuous quality improvement: Impact on process quality and patient safety. Health Care Management Review, 40, 24-34.

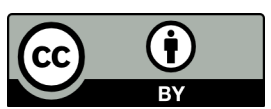

(C) 2021 by the Dr. Jose A. PonceVega and Dr. Irene A. Williams. Submitted for possible open access publication under the terms and conditions of the Creative Commons Attribution (CC BY) license (http://creativecommons.org/licenses/by/4.0/). 